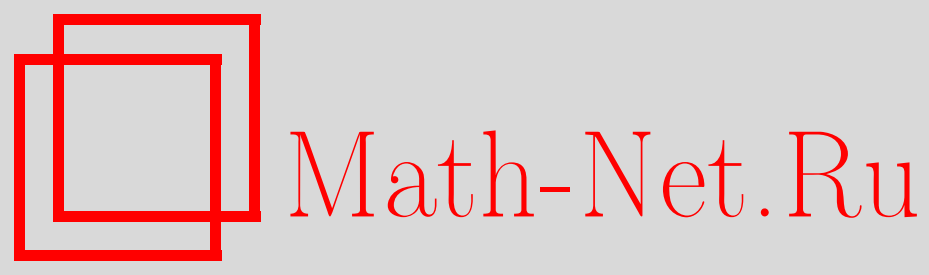

A. А. Досовицкий, Некоторые меры на множестве кусочно-гладких гомеоморфизмов окружности и связанные с ними представления группы диффеоморфизмов окружности, Матем. заметки, 2010, том 88, выпуск 6, 946-949

DOI: https://doi.org/10.4213/mzm8586

Использование Общероссийского математического портала Math-Net.Ru подразумевает, что вы прочитали и согласны с пользовательским соглашением http://www.mathnet.ru/rus/agreement

Параметры загрузки:

IP : 35.173 .219 .149

26 апреля 2023 г., 09:17:41

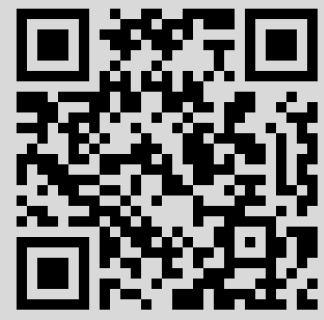




\section{Некоторые меры на множестве кусочно-гладких гомеоморфизмов окружности и связанные с ними представления группы диффеоморфизмов окружности}

\section{А. А. Досовицкий}

1. Введение. В настоящей работе построено семейство мер на некоторых подмножествах множества кусочно-гладких гомеоморфизмов окружности. Доказана их квазиинвариантность относительно действия композицией диффеоморфизмов окружности более высокого класса гладкости. На основе этого получена серия неизвестных ранее неприводимых унитарных представлений группы, сохраняющих фиксированную точку диффеоморфизмов окружности. Развиваются идеи работ Шавгулидзе [1] и Косяка [2], в которых рассматривались меры на множествах диффеоморфизмов отрезка и окружности и соответствующие представления. Близкие методы построения представлений групп диффеоморфизмов использовались в работах Исмагилова [3] (меры на множестве сходящихся последовательностей на окружности) и [4] (пуассоновы меры на пространстве конфигураций, т.е. локально конечных множеств, в $\left.\mathbb{R}^{n}\right)$, а также Вершика, Гельфанда, Граева [5] (меры, в том числе пуассоновы, на пространствах конфигураций на многообразиях).

2. Меры. Определим множества, на которых будут задаваться меры. Каждое такое множество $F\left(t_{1}, \ldots, t_{r}, l_{1}, \ldots, l_{r}\right)$ определяется натуральным числом $r$ и наборами вещественных чисел $\left\{0<t_{1}<\cdots<t_{r}<1\right\}$ и $\left\{l_{1}, \ldots, l_{r}\right\}$. Зафиксируем эти величины. Обозначим через $C_{00}([0,1])$ пространство непрерывных на отрезке $[0,1]$ функций $c(t)$ таких, что $c(0)=c(1)=0$, с нормой $\|c\|_{C}=\sup _{t \in[0,1]}|c(t)|$. Положим $X=X\left(t_{1}, \ldots, t_{r}, l_{1}, \ldots, l_{r}\right)-$ сдвиг пространства $C_{00}([0,1])$ как подмножества в $\mathrm{P} C_{0}([0,1])$ (кусочно-непрерывные на отрезке $[0,1]$ функции $x(t)$ такие, что $x(0)=0)$ на ступенчатую функцию с скачками $l_{j}$ в точках $t_{j}$, т.е. $X=B_{t_{1}, \ldots, t_{r}, l_{1}, \ldots, l_{r}}\left(C_{00}([0,1])\right)$, где

$$
B=B_{t_{1}, \ldots, t_{r}, l_{1}, \ldots, l_{r}}: c(t) \mapsto c(t)+\sum_{j=1}^{r} l_{j} \theta\left(t-t_{j}\right), \quad c \in C_{00}([0,1]), \quad \theta(t)= \begin{cases}1, & t \geqslant 0 \\ 0, & t<0 .\end{cases}
$$

Снабдим $X \ni x, y$ метрикой

$$
r_{X}(x, y)=\sup _{t \in[0,1] \backslash\left\{t_{j}\right\}_{j=1}^{r}}|x(t)-y(t)| .
$$

Обозначим через $\mathrm{P}_{r} \operatorname{Diff}_{+}^{1}([0,1])$ множество кусочно-гладких гомеоморфизмов отрезка $[0,1]$ класса гладкости $C^{1}$, сохраняющих ориентацию и имеющих ровно $r$ изломов, т.е.

$$
\begin{aligned}
\mathrm{P}_{r} \operatorname{Diff} f_{+}^{1}([0,1])=\{f:[0,1] \rightarrow[0,1], f(0)=0, f(1)=1, f \in C([0,1]), & \\
& \exists f^{-1} \in C([0,1]) \exists \tau_{0}=0<\tau_{1}<\cdots<\tau_{r}<1=\tau_{r+1} \\
& \left.f \in C^{1}\left(\left[\tau_{j-1}, \tau_{j}\right]\right), f^{-1} \in C^{1}\left(\left[f\left(\tau_{j-1}\right), f\left(\tau_{j}\right)\right]\right), j=1, \ldots, r\right\} .
\end{aligned}
$$

Зададим отображение $A$ следующей формулой:

$$
(A x)(t)=\frac{\int_{0}^{t} \exp (x(\tau)) d \tau}{\int_{0}^{1} \exp (x(\tau)) d \tau}, \quad\left(A^{-1} f\right)(t)=\ln f^{\prime}(t)-\ln f^{\prime}(0) .
$$


Оно устанавливает взаимно-однозначное соответствие между $\mathrm{P}_{r} C_{0}([0,1])$ (множество кусочно-непрерывных равных нулю в нуле функций на $[0,1]$ с ровно $r$ скачками) и $\mathrm{P}_{r} \operatorname{Diff}_{+}^{1}([0,1])$. Положим $F=F\left(t_{1}, \ldots, t_{r}, l_{1}, \ldots, l_{r}\right)=A X$. Введем на $F$ метрику

$$
r_{F}\left(f_{1}, f_{2}\right)=\sup _{t \in[0,1] \backslash\left\{t_{j}\right\}_{j=1}^{r}}\left|f_{1}^{\prime}(t)-f_{2}^{\prime}(t)\right|
$$

и соответствующую топологию, тогда $A$ будет гомеоморфизмом $X$ в $F$.

Теперь зададим на множестве $F$ меру. Пусть $W_{\sigma}^{0}$ - условная мера Винера (броуновский мост) с дисперсией $\sigma$ в $C_{00}([0,1])$. Борелевскую меру $\mu_{\sigma}$ определим как образ при отображении $A B$ меры $W_{\sigma}^{0}$, т.е. $\mu_{\sigma}(U)=W_{\sigma}^{0}\left((A B)^{-1} U\right)$ для любого борелевского множества $U \subset F$.

Положим

$$
\begin{aligned}
& \operatorname{Diff}_{+, 0}^{k}([0,1])=\left\{f:[0,1] \rightarrow[0,1], f(0)=0, f(1)=1, f \in C^{k}([0,1]),\right. \\
&\left.\exists f^{-1} \in C^{k}([0,1]), f^{(j)}(0)=f^{(j)}(1), j=1, \ldots, k\right\} .
\end{aligned}
$$

Эту группу естественно интерпретировать как группу диффеоморфизмов окружности класса гладкости $C^{k}$, сохраняющих некоторую фиксированную точку. При этом группа $\operatorname{Diff}_{+, 0}^{k}([0,1]) \ni g$ действует на $F \ni f$ композицией: $\left(L_{g} f\right)(t)=g(f(t))$. Несложно показать, что $L_{g} f \in F$ для всех $f \in F$. Следующие теоремы показывают, как преобразуется мера $\mu_{\sigma}$ при этом действии.

Теорема 1. Мера $\mu_{\sigma}$ квазиинвариантна относительно действия $\operatorname{Diff}_{+, 0}^{3}([0,1])$, m.е. для любого борелевского множества $U \subset F$ и любого $g \in \operatorname{Diff}_{+, 0}^{3}([0,1])$ выполняется

$$
\mu_{\sigma}\left(L_{g} U\right)=\int_{U} \rho_{g}^{\sigma}(f) \mu_{\sigma}(d f)
$$

причем плотность $\rho_{g}^{\sigma}$ дается формулой

$$
\begin{gathered}
\rho_{g}^{\sigma}(f)=\frac{1}{g^{\prime}(0)} \exp \frac{1}{\sigma^{2}}\left(\frac{g^{\prime \prime}(0)}{g^{\prime}(0)}\left(f^{\prime}(0)-f^{\prime}(1)\right)+\sum_{j=1}^{r} \frac{g^{\prime \prime}\left(f\left(t_{j}\right)\right)}{g^{\prime}\left(f\left(t_{j}\right)\right)}\left(f^{\prime}\left(t_{j}+0\right)-f^{\prime}\left(t_{j}-0\right)\right)\right. \\
\left.+\int_{0}^{1} S_{g}(f(t))\left(f^{\prime}(t)\right)^{2} d t\right)
\end{gathered}
$$

где

$$
S_{g}(s)=\frac{g^{\prime \prime \prime}(s)}{g^{\prime}(s)}-\frac{3}{2}\left(\frac{g^{\prime \prime}(s)}{g^{\prime}(s)}\right)^{2}
$$

- шварциан функции $g$.

Теорема 2. Мера $\mu_{\sigma}$ квазиинвариантна относителъно действия $\operatorname{Diff}_{+, 0}^{2}([0,1])$.

ЗАмЕЧАниЕ 1 . Чтобы получить плотность меры $\mu_{\sigma}$ относительно действия диффеоморфизма $h \in \operatorname{Diff}_{+, 0}^{2}([0,1])$, аппроксимируем его последовательностью $g_{n} \in \operatorname{Diff}_{+, 0}^{4}([0,1])$ так, чтобы

$$
\sup _{t \in[0,1]}\left|h^{\prime \prime}(t)-g_{n}^{\prime \prime}(t)\right| \stackrel{n \rightarrow+\infty}{\longrightarrow} 0 .
$$

Оказывается, что тогда последовательность плотностей $\rho_{g_{n}}^{\sigma}(f)$ сходится по мере $\mu_{\sigma}$ к некоторой измеримой функции, которая и будет плотностью при действии $h$. Близкие вопросы изучаются в работе Кузьмина [6] методами теории случайных процессов. 
3. Представления. Для каждого $\lambda \in \mathbb{R}$ зададим унитарное представление группы $\operatorname{Diff}_{+, 0}^{3}([0,1])$ в пространстве $L_{2}\left(F, \mu_{\sigma}\right) \ni \Phi$ квадратично интегрируемых по мере $\mu_{\sigma}$ комплекснозначных функций на $F$ следующей формулой:

$$
\left(U_{g}^{\sigma, \lambda} \Phi\right)(f)=\left(\rho_{g^{-1}}(f)\right)^{1 / 2+\lambda \imath} \Phi\left(g^{-1} \circ f\right),
$$

где $\imath$ - мнимая единица. Следующие теоремы описывают основные свойства этих представлений.

Теорема 3. Все представления $U^{\sigma, \lambda}$ неприводимы.

Теорема 4. Пусть $U_{1}^{\sigma_{1}, \lambda_{1}}$ и $U_{2}^{\sigma_{2}, \lambda_{2}}$ - описанные выше представления $\operatorname{Diff}_{+, 0}^{3}([0,1])$ в пространствах $L_{2}\left(F\left(t_{1}^{1}, \ldots, t_{r_{1}}^{1}, l_{1}^{1}, \ldots, l_{r_{1}}^{1}\right), \mu_{\sigma_{1}}^{1}\right)$ u $L_{2}\left(F\left(t_{1}^{2}, \ldots, t_{r_{2}}^{2}, l_{1}^{2}, \ldots, l_{r_{2}}^{2}\right), \mu_{\sigma_{2}}^{2}\right)$ coответственно. Они эквивалентны тогда и только тогда, когда выполняются следующие равенства:

$$
r_{1}=r_{2}, \quad \lambda_{1}=\lambda_{2}, \quad \sigma_{1}=\sigma_{2}, \quad t_{j}^{1}=t_{j}^{2}, \quad l_{j}^{1}=l_{j}^{2}, \quad j=1, \ldots, r_{1} .
$$

ЗАмечАние 2. При построении меры в начале п. 2 можно было не требовать равенства $c(1)=0$ и воспользоваться обыкновенной мерой Винера на $C_{0}([0,1])$ вместо броуновского моста на $C_{00}([0,1])$. Однако представления группы диффеоморфизмов окружности с фиксированной точкой $\operatorname{Diff}_{+, 0}^{3}([0,1])$ в пространстве квадратично интегрируемых по так построенной мере функций были бы приводимы. Таким образом, дополнительное ограничение $c(1)=0$ оправдывается тем, что оно упрощает структуру представлений группы $\operatorname{Diff}_{+, 0}^{3}([0,1])$.

4. Идеи доказательств. Теорема 1 доказывается путем приближения броуновского моста на пространстве $C_{00}([0,1])$ конечномерными цилиндрическими мерами, непосредственного вычисления их плотностей при действии группы Diff $+, 0,0([0,1])$ и затем перехода к пределу по размерности цилиндрических мер. Основные моменты повторяют доказательство теоремы 1 в [1]. Вывод теоремы 2 описан в замечании 1 . Технически он осуществляется переходом к конечномерным приближениям и далее с помощью суммирования по частям, неравенства Чебышева и свойств броуновского моста.

Доказательства теорем 3 и 4 проводятся для унитарно эквивалентных представлениям $U^{\sigma, \lambda}$ представлений $V^{\sigma, \lambda}$ в пространстве $\mathscr{H}=L_{2}\left(X, B W_{\sigma}^{0}\right)$, где $B W_{\sigma}^{0}$ означает образ броуновского моста в $C_{00}([0,1])$ при отображении $B$ (см. (1)). Везде далее $\mathscr{H}$ будет обозначать именно это конкретное пространство. Представления $V^{\sigma, \lambda}$ получаются из представлений $U^{\sigma, \lambda}$ заменой аргумента посредством оператора $A$ (см. (2)). Доказательства основываются на известном утверждении из теории представлений в гильбертовых пространствах и двух леммах.

ПредЛОЖЕНИЕ 1. Пусть $V$ - унитарное представление группы $G$ в произвольном комплексном гильбертовом пространстве $H$. Тогда неприводимость представления $V$ равносильна следующему утверждению: для любого линейного непрерывного оператора $S: H \rightarrow H$ из того, что $S V_{g}=V_{g} S$ для всех $g \in G$, следует, что $S=\lambda \cdot \mathrm{id}, \lambda \in \mathbb{C}$.

Обозначим $R_{a}$ - оператор поточечного умножения на функцию $a(x)$ в пространстве $\mathscr{H}$.

Лемма 1. Пусть $S: \mathscr{H} \rightarrow \mathscr{H}$ - линейный непрерывный оператор такой, что

$$
S V_{g}^{\sigma, \lambda}=V_{g}^{\sigma, \lambda} S \quad \text { для всех } g \in \operatorname{Diff}_{+, 0}^{3}([0,1]) .
$$

Тогда для всех $\alpha \in \mathbb{R}, t \in[0,1]$ для функиий

$$
b_{\alpha, t}^{\sigma, \lambda}(x)=\exp \left(-\frac{\left(1+4 \lambda^{2}\right) \alpha^{2}}{8 \sigma^{2} \varphi_{x}^{\prime}(t)}\right), \quad \varphi_{x}=(A x)^{-1},
$$

выполняется $S R_{b_{\alpha, t}^{\sigma, \lambda}}=R_{b_{\alpha, t}^{\sigma, \lambda}} S$. 
Лемма 2. Пусть $S: \mathscr{H} \rightarrow \mathscr{H}$ - линейный непрерывный оператор такой, что

$$
S R_{b_{\alpha, t}^{\sigma, \lambda}}=R_{b_{\alpha, t}^{\sigma, \lambda}} S \quad \text { для всех } \quad \alpha \in \mathbb{R}, \quad t \in[0,1] .
$$

Тогда $S R_{a}=R_{a} S$ для любой непрерывной ограниченной функиии а $\in \mathscr{H}$.

Лемма 1 доказывается приближением операторов $R_{b_{\alpha, t}^{\sigma, \lambda}}$ операторами $V_{g}^{\sigma, \lambda}$, лемма $2-$ приближением операторов $R_{a}$ операторами $R_{b_{\alpha, t}^{\sigma, \lambda}}$.

Пусть $\mathbf{1}(x)$ - тождественно единичная функция в $\mathscr{H}$. Обозначим $F_{0}=S \mathbf{1}$. Тогда из леммы 2 , теоремы 2 , коммутирования $S$ с операторами представления $V_{g}^{\sigma, \lambda}$ и эргодичности меры Винера относительно сдвигов можно вывести, что $F_{0}=$ const $=c$. Значит, $S a=S R_{a} \mathbf{1}=R_{a} S \mathbf{1}=c a$ и по предложению 1 теорема 3 доказана.

Утверждение теоремы 4 выводится от противного: предположение о существовании сплетающего оператора между представлениями $V_{1}^{\sigma_{1}, \lambda_{1}}$ и $V_{2}^{\sigma_{2}, \lambda_{2}}$, т.е. такого непрерывного линейного оператора

$$
S: L_{2}\left(X\left(t_{1}^{1}, \ldots, t_{r_{1}}^{1}, l_{1}^{1}, \ldots, l_{r_{1}}^{1}\right), B W_{\sigma_{1}}^{0}\right) \rightarrow L_{2}\left(X\left(t_{1}^{2}, \ldots, t_{r_{2}}^{2}, l_{1}^{2}, \ldots, l_{r_{2}}^{2}\right), B W_{\sigma_{2}}^{0}\right),
$$

что

$$
S V_{1, g}^{\sigma_{1}, \lambda_{1}}=V_{2, g}^{\sigma_{2}, \lambda_{2}} S \quad \text { для всех } \quad g \in \operatorname{Diff}_{+, 0}^{3}([0,1]),
$$

приводится сначала к равенству $S R_{b_{\alpha, t}^{\sigma_{1}, \lambda_{1}}}=R_{b_{\alpha, t}^{\sigma_{2}, \lambda_{2}}} S$ (аналогично лемме 1 ), а затем к противоречию манипуляциями над обеими частями последнего равенства.

В заключение автор выражает искреннюю благодарность Е. Т. Шавгулидзе за постановку задачи, плодотворные обсуждения и помощь в подготовке статьи.

\section{СПИСОК ЦИТИРОВАННОЙ ЛИТЕРАТУРЫ}

[1] E. T. Shavgulidze, Russ. J. Math. Phys., 7:4 (2000), 464-472. [2] A. V. Kosyak, J. Funct. Anal., 125:2 (1994), 493-547. [3] Р. С. Исмагилов, Функи. анализ и его прил., 5:3 (1971), 45-53. [4] Р. С. Исмагилов, Матем. сб., 98:1 (1975), 55-71. [5] А. М. Вершик, И. М. Гельфанд, М.И. Граев, УМH, 30:6 (1975), 3-50. [6] P. A. Kuzmin, J. Math. Anal. Appl., 330:1 (2007), 744-750.

\section{А. А. Досовицкий}

Поступило Московский государственный университет

им. М. В. Ломоносова

E-mail: adosovitskiy@gmail.com 